\title{
Mental disorders in new parents before and after birth: a population-based cohort study
}

\author{
Fenglian Xu, Elizabeth Sullivan, Colin Binns and Caroline S.E. Homer
}

\section{Background}

Mental disorders of women during the postnatal period are a major public health problem. Compared with women's mental disorders, much less attention has been paid to men's mental disorders in the perinatal period. To date, there have been no reports in the literature describing secular changes of both maternal and paternal hospital admissions for mental disorders over the period covering the year before pregnancy (nonparents), during pregnancy (expectant parents) and up to the first year after birth (parents) based on linked parental data. The co-occurrences of couples' hospital admissions for mental disorders have not previously been investigated.

\section{Aims}

To describe maternal and paternal hospital admissions for mental disorders before and after birth. To compare the co-occurrences of parents' hospital admissions for mental disorder in the perinatal period.

\section{Method}

This is a cohort study using paired parents' population data from the New South Wales (NSW) Perinatal Data Collection (PDC), Registry of Births, Deaths and Marriages (RBDM) and Admitted Patients Data Collection (APDC). The study included all parents ( $n=196669$ couples) who gave birth to their first child in NSW between 1 January 2003 and 31 December 2009.

\section{Results}

The hospital admission rate for women with a principal mental disorder diagnosis in the period between the year before pregnancy and the first year after birth was significantly higher than that for men. Parents' mental disorders influenced each other. If a man was admitted to hospital with a principal mental disorder diagnosis, his wife or partner was more likely to be admitted to hospital with a principal mental disorder diagnosis compared with women whose partner had not had a hospital admission, and vice versa.

\section{Conclusions}

Mothers' mental disorders after birth increased more significantly than fathers. However, fathers' mental disorders significantly impacted the co-occurrence of mothers' mental disorders.

\section{Declaration of interest}

None.

\section{Copyright and usage}

(C) The Royal College of Psychiatrists 2016. This is an open access article distributed under the terms of the Creative Commons Non-Commercial, No Derivatives (CC BY-NC-ND) licence.
Mental disorders of women during the postnatal period are a major public health problem that can significantly impact on the health of the whole family. ${ }^{1-3}$ There are consistent reports of an increase in hospital admissions for mental disorders in the first year after birth. ${ }^{4-7}$ A recent prospective cohort study in Melbourne $(n=1507$ nulliparous women) found that $16.1 \%$ of women reported depressive symptoms during the first 12 months post-partum. ${ }^{8}$ In New South Wales (NSW), a population-based study found that the hospital admission rates for psychiatric disorders in the first year after birth increased significantly between 2001 and 2010 with a more marked increase from 2005 (1.16\% in 2001, 2.28\% in 2010). ${ }^{9}$ However, the studies on women during pregnancy is less consistent. ${ }^{5,6,10,11}$ The studies based on health service data showed a decrease in hospital admission for mental disorders during pregnancy. ${ }^{6,7}$ The studies based on surveys reported an increase ${ }^{11,12}$ or a decrease ${ }^{5}$ in women's mental disorders during pregnancy.

Compared with maternal depression, much less attention has been paid to men's mental disorders in the perinatal period. ${ }^{13}$ Studies of fathers' hospital admissions for mental disorders during the perinatal period have shown varying findings. ${ }^{6,14}$ A systematic review showed that the rate of diagnosed depression in new fathers at 6 weeks post-partum was around $2-5 \% .{ }^{15}$ An integrative review from community samples showed that the incidence of paternal depression ranged from 1.2 to $25.5 \%$ during the first post-partum year. ${ }^{13}$ The range was very wide because of differences in the measuring tools used, cut-off point, time of measurement and study population. A study in England $(n=7018$ partners of women in the Avon Longitudinal Study of Pregnancy and Childhood) showed that the prevalence of paternal depression (Edinburgh Postnatal Depression Scale (EPDS) Scores >12) was $3.5 \%$ at 18 weeks gestation and $3.3 \%$ at 8 weeks following the birth. ${ }^{16}$ A cohort study based on Danish population data (630 373 women and 547431 men) showed that new fathers had a decreased risk of hospital admission for mental disorders in the first year after birth compared with non-fathers. ${ }^{6}$

There are relatively few studies focusing on the association between paternal and maternal mental disorders. ${ }^{17}$ A study based on community data in England found that men's depressive symptoms were correlated with their partners' depressive symptoms before and after birth. ${ }^{16}$ An integrative review showed that father's depression following the birth was associated with his partner's depression in pregnancy and after birth. ${ }^{18}$ Another integrative review reported that the incidence of paternal depression were higher in men whose partners were experiencing postpartum depression. ${ }^{13}$

To date, there have been no reports in the literature describing secular changes of both maternal and paternal hospital admissions for mental disorders over the period covering the year before pregnancy (non-parents), during pregnancy (expectant parents) and up to the first year after birth (parents) based on linked parental data. The co-occurrences of couples' hospital admissions for mental disorders have not previously been investigated. Therefore, the aims of this study were to use linked population data from Australia's most populous state (New South Wales) to: (a) describe maternal and paternal hospital admissions for mental disorders before pregnancy, during pregnancy and after birth; and 
(b) compare the co-occurrence of parents' hospital admissions for mental disorders in the perinatal period.

\section{Method}

\section{Study population and design}

This is a population-based cohort study using linked data from the NSW Perinatal Data Collection (PDC), NSW Registry of Births, Deaths and Marriages (RBDM) and the NSW Admitted Patients Data Collection (APDC). The study included all parents who gave birth to their first child in NSW between 1 January 2003 and 31 December 2009. The details of the study population and data linkage are described in Fig. 1.

The mother's PDC birth records, which included mother's and baby's Project Person Number (PPN), were linked with RBDM which included baby's, mother's and father's PPN. The linked PDC-RBDM birth records between 1 January 2003 and 31 December 2009 were linked with parents' APDC records between 1 January 2001 and 31 December 2010, so that hospital admissions for these parents could be traced back for their pregnancy period (expectant parents), the year before pregnancy (nonparents) and followed up 1 year after birth (parents). The couples were followed up over the three periods from the year before pregnancy, pregnancy to the last month of the first year after birth. The APDC records selection and data linkage are detailed in Fig. 2. Parents' hospital admissions for mental disorders were identified by APDC records.

The PDC is a population-based surveillance system that includes all births of at least 20 weeks gestation or at least $400 \mathrm{~g}$ birthweight in NSW. It includes all births in public and private hospitals as well as home births, and includes information on maternal characteristics, pregnancy, labour, delivery and neonatal outcomes. The RBDM is a database of birth registrations. Under the Births, Deaths and Marriages Registration Act 1995, all babies in NSW must be registered within 60 days of birth, ${ }^{19}$ and the Registry therefore includes babies' and their parents' information such as age and place of residence. The APDC is a routinely collected census of all hospital separations. It includes all patient hospitalisations in NSW public and private hospitals including psychiatric hospitals and admissions for same day procedures. It includes information on patient demographics, diagnoses and clinical procedures. Since 1999, the diagnoses for admissions have been coded according to the 10th revision of the International Statistical Classification of Diseases and Related Health Problems, Australian Modification (ICD-10-AM). ${ }^{20}$

The data linkage was performed by the NSW Department of Health Centre for Health Record Linkage (CHeReL) using probabilistic record linkage methods and ChoiceMaker software. ${ }^{21}$ Identifying information from PDC and APDC data sets was included in the Master Linkage Key constructed by the CHeReL. At the completion of the process, each record was assigned a Person Project Number (PPN) to allow records for the same individual to be linked. Based on the 1000 randomly selected sample of records, the false positive rate of the linkage was $0.3 \%$ and false negative $<0.5 \%$.

\section{Definitions}

The principal diagnosis refers to the diagnosis which was chiefly responsible for APDC hospital admission. ${ }^{22}$ Mental disorders refer to the principal diagnoses for psychiatric disorders and disorders due to substance use. The first hospital admission refers to the

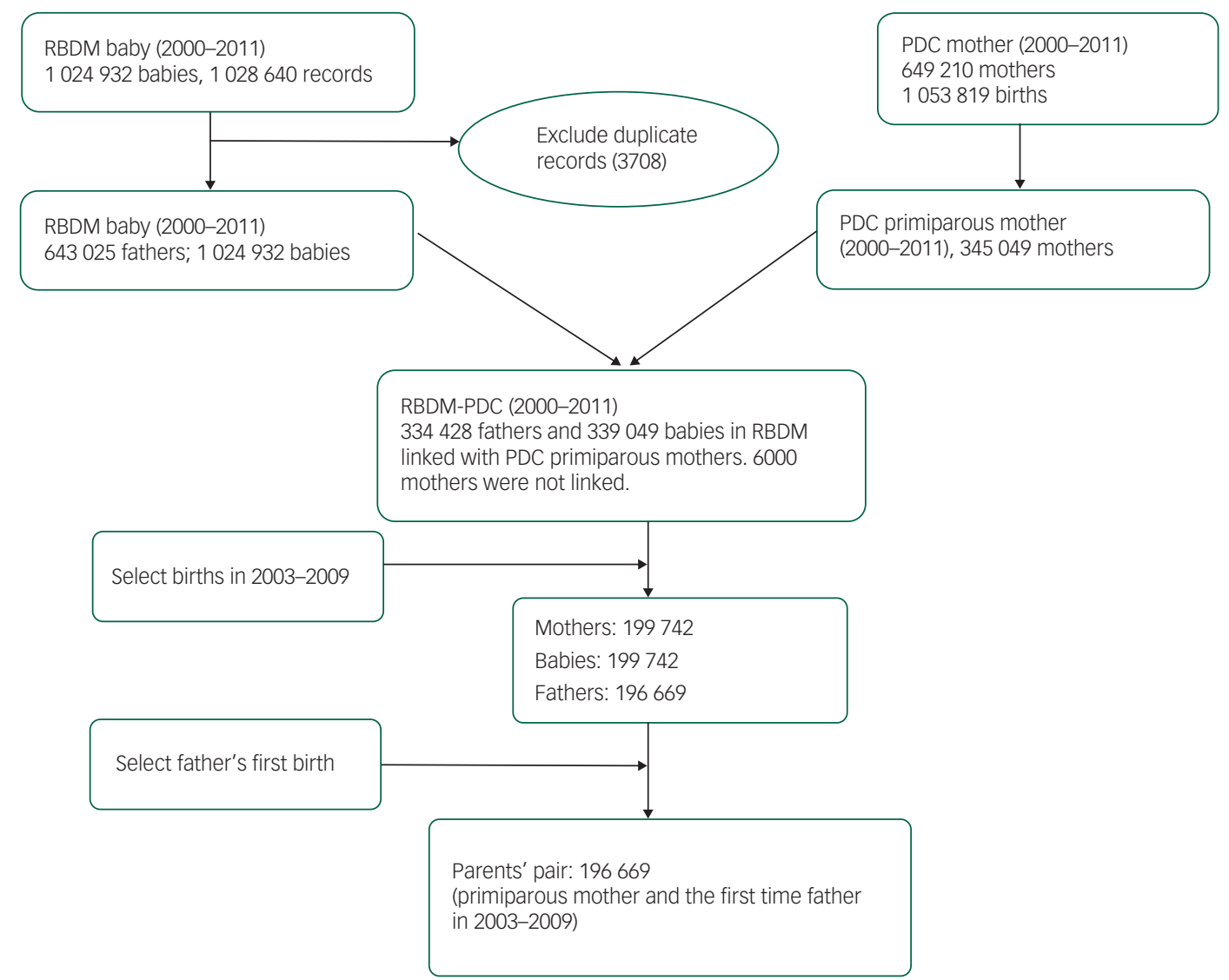

Fig. 1 The data linkage and study population. RBDM, New South Wales registry of births, deaths and marriages; PDC, New South Wales perinatal data collection. 


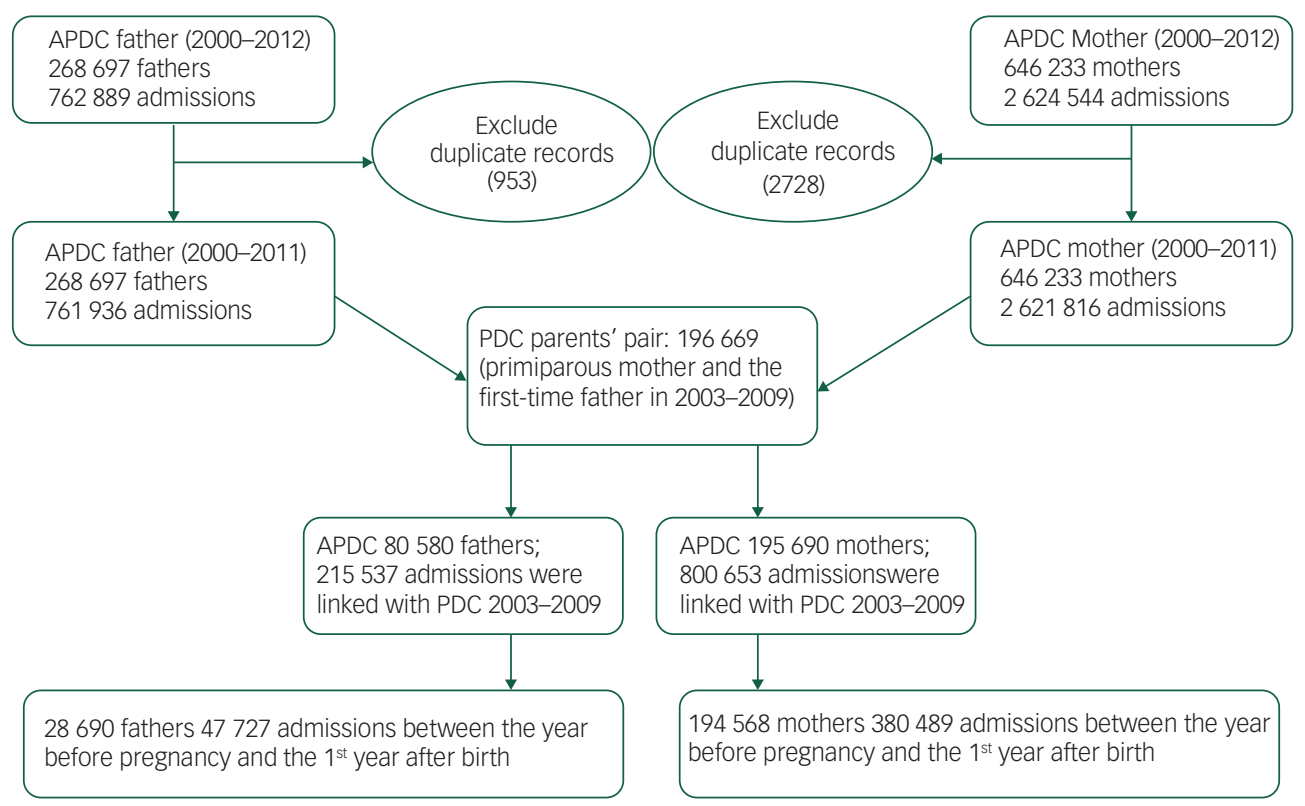

Fig. 2 Parents' hospital admission data linked with birth data. APDC, New South Wales admitted patients data collection.

first hospital admission between the first month of the year before pregnancy and the last month of the year after birth.

All hospital admissions include the first hospital admission and re-admissions in the study periods. Non-parents, expectant parents and parents: the couples in the year before their first pregnancy were regarded as non-parents, during pregnancy as expectant parents and the first year after birth as the parents.

\section{Diagnosis of mental disorders}

The diagnoses for each admission in this study have been coded according to the Australian modification to the World Health Organization ICD-10 Classification of Diseases and Related Health Problems (ICD-10-AM).$^{20}$ Parents with mental disorders were identified using ICD-10-AM diagnosis codes: (1) F10-19 [mental and behaviour disorders due to use of alcohol and other substances]; (2) F32-33 [depressive disorder]; (3) F53 [mental and behavioural disorders associated with the puerperium]; (4) F41 and F43 [anxiety and adjustment disorders]; (5) F20 and F31 [schizophrenia and bipolar affective disorders]; (6) others referred to the rest F codes; (7) F00-99 [overall mental disorders].

In this study, only the hospital admission with a principal diagnosis of a mental disorder between the year before pregnancy and the first year after birth was included in the analysis.

\section{Statistical analysis}

Descriptive statistics were used to analyse the rate of hospital admission (including the 95\% confidence interval, CI), parents' age and mothers' characteristics. Person-year was used as the denominator for the analysis of rates to allow comparison between different periods or between maternal and paternal populations. The analyses were conducted using IBM SPSS (Statistical Package for Social Science) Statistics version $22 .^{23}$

\section{Results}

The study population were primiparous women who gave birth in NSW between 2003 and 2009 and their partners. First, primiparous mothers were selected from PDC birth records. Then the women's PDC birth records were linked with their babies' RBDM records.
Finally the births between 2003 and 2009 were selected. There were 199742 primiparous women who met the inclusion criteria and were linked with their partners through their babies' RBDM records. Of the 199742 couples, there were 196669 fathers (98.46\%) who had first births. A total of 196669 couples were included in the data analysis (Fig. 1). Women's characteristics and demographic factors are described in Table 1. The fathers (median 31.56 , mean 31.95 , standard deviation (s.d.) $=6.64$, range $13.81-$ 82.80 ) were 3 years older than the mothers (median 29.02, mean 28.49 , s.d. $=5.78$, range $12.01-56.04)(P<0.05)$.

Table 2 shows the first hospital admission rates of the couples for the principal diagnoses of mental disorders from the year before pregnancy up to the last month of the first year after birth in NSW between 2003 and 2009. Women's first hospital admission rate for the principal diagnoses of mental disorders was significantly higher than men (Table 2$)(P<0.05)$. In the 196669 couples, 4896 women (8.87 per 1000 person-year, 95\% CI 8.629.12) were admitted to a hospital for the principal diagnoses of a mental disorder in the period between the year before pregnancy and the last month of the first year after birth. There were 1287 men (2.33 per 1000 person-year, 95\% CI 2.20-2.46) who were admitted to a hospital for the principal diagnoses of a mental disorder in the same period. The women's hospital admission rate with a mental disorder was 3.81 times greater than the men's (Table 2).

In the three study periods, the maternal first hospital admission rate for the principal diagnoses of mental disorders was the highest after birth (rate 18.91 per 1000 person-year, 95\% CI 18.31-19.51) compared with the year before pregnancy (rate 4.68 per 1000 person-year, 95\% CI 4.38-4.98) and during pregnancy (rate 1.61 per 1000 person-year, 95\% CI 1.41-1.81). For the diagnoses of depressive disorders (F32-33), anxiety and adjustment disorders (F41 and F43), the pattern was similar; the hospital admission rate was the highest in the period after birth, and lowest in pregnancy $(P<0.05)$. The first hospital admission rate for the diagnosis of mental and behaviour disorders associated with puerperium (F53) was 5.35 per 1000 person-year (95\% CI 5.03-5.67), one year after birth. For the diagnosis of mental and behaviour disorders due to use of alcohol and other substances (F10-19), maternal hospital admission rate in the year 
Table 1 New mother's characteristics in NSW, Australia, 2003-2009

\begin{tabular}{|c|c|c|c|}
\hline & $n$ & $\%$ & $95 \% \mathrm{Cl}$ \\
\hline \multicolumn{4}{|l|}{ Maternal age, years* } \\
\hline$<20$ & 14001 & 7.12 & $7.01-7.23$ \\
\hline $20-24$ & 35979 & 18.3 & $18.13-18.47$ \\
\hline $25-29$ & 59696 & 30.36 & $30.16-30.56$ \\
\hline 30-34 & 57448 & 29.21 & $29.01-29.41$ \\
\hline $35-39$ & 24167 & 12.29 & $12.14-12.44$ \\
\hline $40-44$ & 5065 & 2.58 & $2.51-2.65$ \\
\hline $45+$ & 291 & 0.15 & $0.13-0.17$ \\
\hline Missing & 22 & 0.01 & \\
\hline Total & 196669 & 100 & \\
\hline \multicolumn{4}{|l|}{ Woman's country of birth* } \\
\hline Australia & 133085 & 67.67 & $67.46-67.88$ \\
\hline Others countries & 63584 & 32.33 & $32.00-33.00$ \\
\hline Total & 196669 & 100 & \\
\hline \multicolumn{4}{|l|}{ Remoteness* } \\
\hline Major cities & 137243 & 70.7 & $70.50-70.90$ \\
\hline Inner regional & 43086 & 22.19 & $22.01-22.37$ \\
\hline Out regional and remote & 13803 & 7.11 & $7.00-7.22$ \\
\hline Missing & 2537 & 1.3 & $1.25-1.35$ \\
\hline Total & 196669 & 100 & \\
\hline \multicolumn{4}{|l|}{ Smoking during pregnancy* } \\
\hline No & 175107 & 89.31 & $89.17-89.45$ \\
\hline Yes & 20951 & 10.69 & $10.55-10.83$ \\
\hline Missing & 611 & 0.31 & $0.29-0.33$ \\
\hline Total & 196669 & 100 & \\
\hline \multicolumn{4}{|c|}{ Index of Relative SE Disadvantage Quintile* } \\
\hline Least disadvantaged & 46102 & 23.75 & $23.56-23.94$ \\
\hline 2 & 40650 & 20.94 & $20.76-21.12$ \\
\hline 3 & 36778 & 18.94 & 18.77-19.11 \\
\hline 4 & 33424 & 17.22 & $17.05-17.39$ \\
\hline Most disadvantaged & 37178 & 19.15 & $18.98-19.32$ \\
\hline Missing & 2537 & 1.29 & $1.24-1.34$ \\
\hline Total & 196669 & 100 & \\
\hline \multicolumn{4}{|l|}{ Mode of birth } \\
\hline Vaginal & 134078 & 68.21 & $68.00-68.42$ \\
\hline Caesarean section & 62484 & 31.79 & $31.58-32.00$ \\
\hline Missing & 107 & 0.05 & $0.04-0.06$ \\
\hline Total & 196669 & 100 & \\
\hline \multicolumn{4}{|l|}{ Maternal diabetes mellitus* } \\
\hline No & 195629 & 99.47 & $99.44-99.50$ \\
\hline Yes & 1040 & 0.53 & $0.50-0.56$ \\
\hline Total & 196669 & 100 & \\
\hline \multicolumn{4}{|l|}{ Gestational diabetes } \\
\hline No & 187587 & 95.38 & $95.29-95.47$ \\
\hline Yes & 9082 & 4.62 & $4.53-4.71$ \\
\hline Total & 196669 & 100 & \\
\hline \multicolumn{4}{|l|}{ Maternal hypertension* } \\
\hline No & 194833 & 99.07 & $99.03-99.11$ \\
\hline Yes & 1836 & 0.93 & $0.89-0.97$ \\
\hline Total & 196669 & 100 & \\
\hline
\end{tabular}

before pregnancy was higher than the periods in pregnancy and after birth $(P<0.05)$. For schizophrenia and bipolar affective disorders (F20, F31), maternal hospital admission rate in pregnancy was lower than the year before pregnancy and the year after birth (Table 2 and Fig. 4) $(P<0.05)$.

The paternal first hospital admission rate for the principal diagnosis of mental disorders in pregnancy (rate 1.64 per 1000 person-year, 95\% CI 1.44-1.84) was lower than the year before pregnancy (rate 2.66 per 1000 person-year, 95\% CI 2.43-2.89) and the first year after pregnancy (rate 2.56 per 1000 person-year, 95\% CI 2.34-2.78). Similar to women, paternal hospital admissions for anxiety and adjustment disorders (F41, F43) after birth increased significantly compared with the time periods before birth
$(P<0.05)$. The rate change however was significantly less than for women $(P<0.05)$. For the diagnosis of mental and behaviour disorders due to use of alcohol and other substances (F10-19) and depressive disorders (F32-33), paternal hospital admission in pregnancy was lower than the year before pregnancy and the first year after birth $(P<0.05)$. For schizophrenia and bipolar affective disorders (F20, F31), there was no significant difference in paternal hospital admission rates among the three time periods (the year before pregnancy, pregnancy and the first year after birth) (Table 2 and Fig. 5).

The maternal first hospital admission rates in the year before pregnancy and especially in the first year after birth were significantly higher than paternal first hospital admission rates 


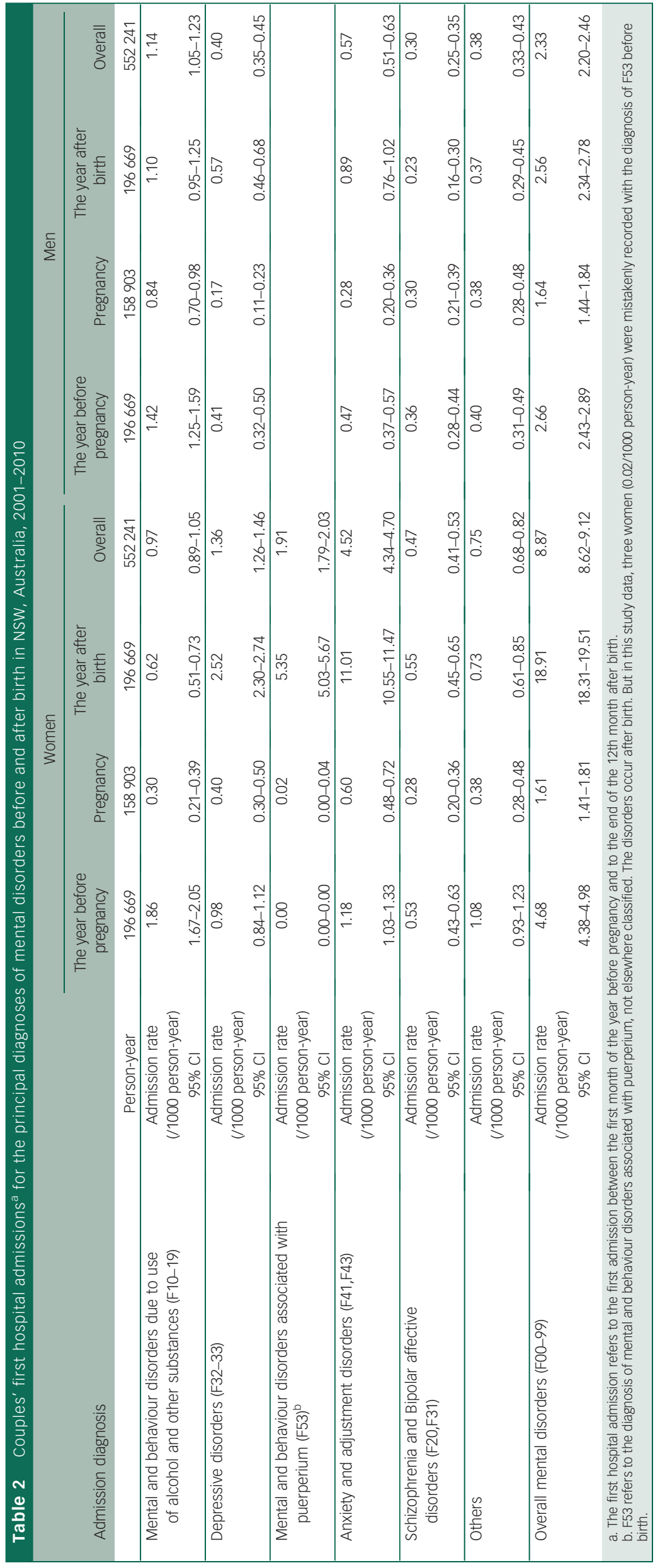




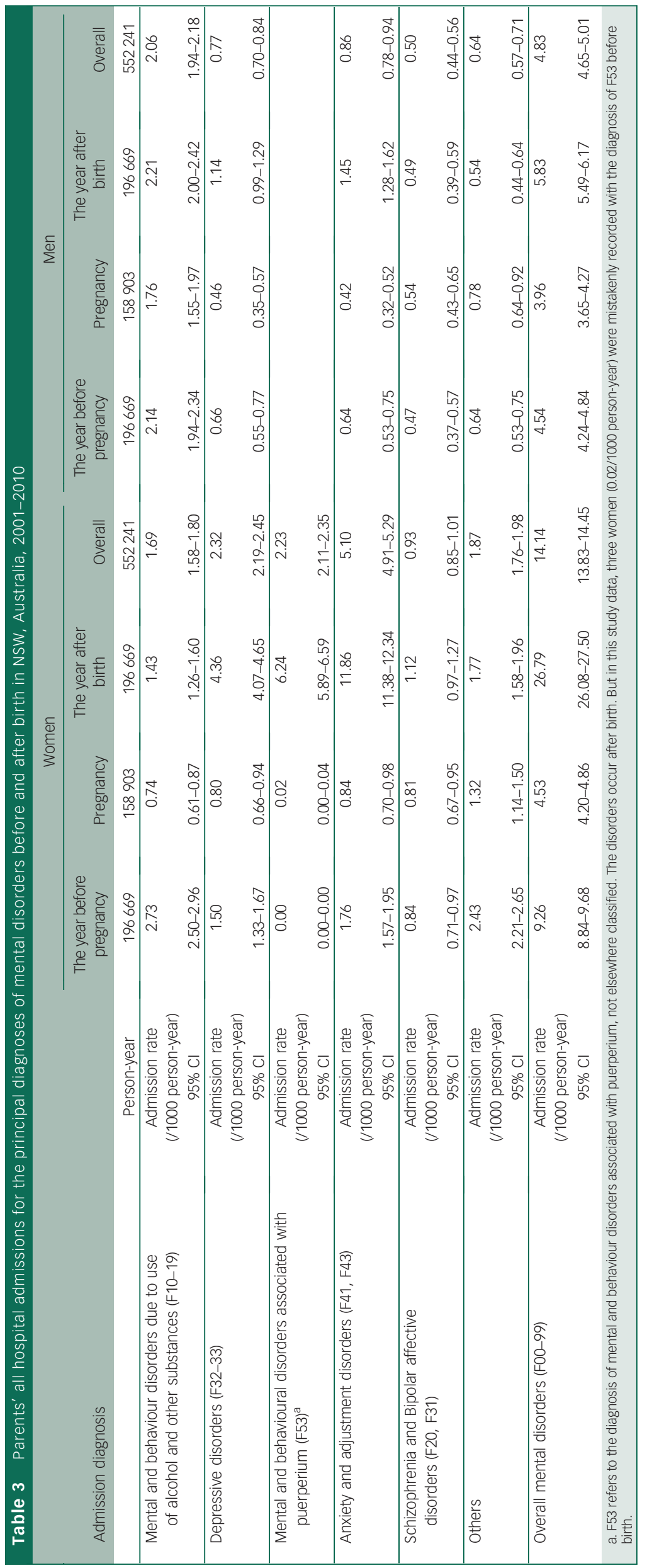


$(P<0.05)$. There was no significant difference between maternal and paternal first hospital admission rates during the period of pregnancy (Table 2).

The rate of all hospital admissions of the couples for mental disorders principal diagnoses between the year before pregnancy and the first year after birth were described in Table 3. The maternal hospital admission rate $14.14 / 1000$ person-year (95\% CI 13.83-14.45) which was significantly higher (rate ratio 2.93 times) than the paternal hospital admission rate (rate $4.83 / 1000$ personyear, 95\% CI 4.65-5.01). The change for all hospital admissions with mental disorders principal diagnoses over the periods before and after birth was similar to the first hospital admissions (Table 2). The maternal hospital admission rates in the year before pregnancy and especially in the first year after birth were significantly higher than paternal hospital admission rates $(P<0.05)$. There was no significant difference between maternal and paternal hospital admission rates during the period of pregnancy (Table 3, Figs. 4 and 5).

Figure 3 shows the distribution of the maternal and paternal hospital admissions for the principal diagnosis of mental disorders during the three time periods (the year before pregnancy, pregnancy and the first year after birth). Women's hospital admissions, including first and all principal diagnoses for mental disorders, increased significantly after giving birth and peaked in the 3rd and 4th month after birth (maternal first hospital admission rate 29.84 per 1000 person-year, 95\% CI 28.00-31.68; maternal all hospital admission rate 38.20 per 1000 person-year, 95\% CI 36.12-40.28 in month 3-4 after birth) (Fig. 3). Compared with women, men's hospital admission rates were lower except during the period of pregnancy (Tables 2 and 3) and did not have the same peak in the $3 \mathrm{rd}$ and 4 th month after birth.

Figure 4 shows that maternal hospital admissions for mental disorders were mainly attributed to anxiety and adjustment disorders (F41 and F43); mental and behaviour disorders associated with puerperium (F53) and depressive disorders (F32-33).

Figure 5 showed that paternal hospital admissions for mental disorders were mainly attributed to mental and behavioural disorders due to use of alcohol and other substances (F10-19); anxiety and adjustment disorders (F41 and F43) and depressive disorders (F32-33).

Table 4 shows the co-occurrence of mother's first hospital admissions for principal diagnoses of mental disorders with fathers. If a man was admitted to a hospital with a principal diagnosis of a mental disorder, his partner was more likely to be admitted to a hospital with a principal diagnosis of a mental disorder (women's hospital admission rate 12.03, 95\% CI 10.2113.85), particularly in the period after birth, compared with the women whose partner did not have the hospital admission (women's hospital admission rate $0.86,95 \%$ CI $0.78-0.94$ ).

Table 5 shows the co-occurrence of father's first hospital admissions for principal mental disorder diagnoses with mothers. If a woman was admitted to a hospital with a principal diagnosis of mental disorder, her partner was also more likely to be admitted to a hospital with a principal diagnosis of a mental disorder (men's hospital admission rate 3.04, 95\% CI 2.56-3.52), particularly in the period before pregnancy, compared with men whose partner did not have a hospital admission for a mental disorder (men's hospital admission rate $0.21,95 \%$ CI $0.17-0.25$ ).

Women were more likely to be impacted by their partner's mental health problems compared with men (Tables 4 and 5) $(P<0.05)$. In other words, a man's mental disorders impacted his partner's hospital admissions for mental disorders more significantly than woman's mental disorders on her partner's (Tables 4 and 5).

\section{Discussion}

This study provides a complete picture of couple's first hospital admissions for principal diagnoses of mental disorders during the year before pregnancy (non-parents), pregnancy (expectant parents) and the first year after birth (parents). Compared with nonmothers, mothers had significantly more hospital admissions for mental disorder diagnoses (mother's first hospital admission rate 18.91 per 1000 person-year, 95\% CI 18.31-19.51; non-mother's first hospital admission rate 4.68 per 1000 person-year, 95\% CI 4.38-4.98). Compared with non-fathers, fathers did not have the increased risk for hospital admissions for mental disorders (father's first hospital admission rate 2.56 per 1000 person-year, 95\% CI 2.34-2.78; non-father's first hospital admission rate 2.66 per 1000 person-year, 95\% CI 2.43-2.89). Both expectant parents were less likely to be admitted to hospitals for mental disorder diagnoses compared with non-parents (expectant mother's first hospital admission rate 1.61 per 1000 person-year, 95\% CI 1.411.81; expectant father's first hospital admission rate 1.64 per 1000 person-year, 95\% CI 1.44-1.84). The non-mother's first hospital admission rate for mental disorders was 1.76 times higher than non-fathers. The mother's first hospital admission rate for mental disorders was 7.39 times higher than that for fathers. There was

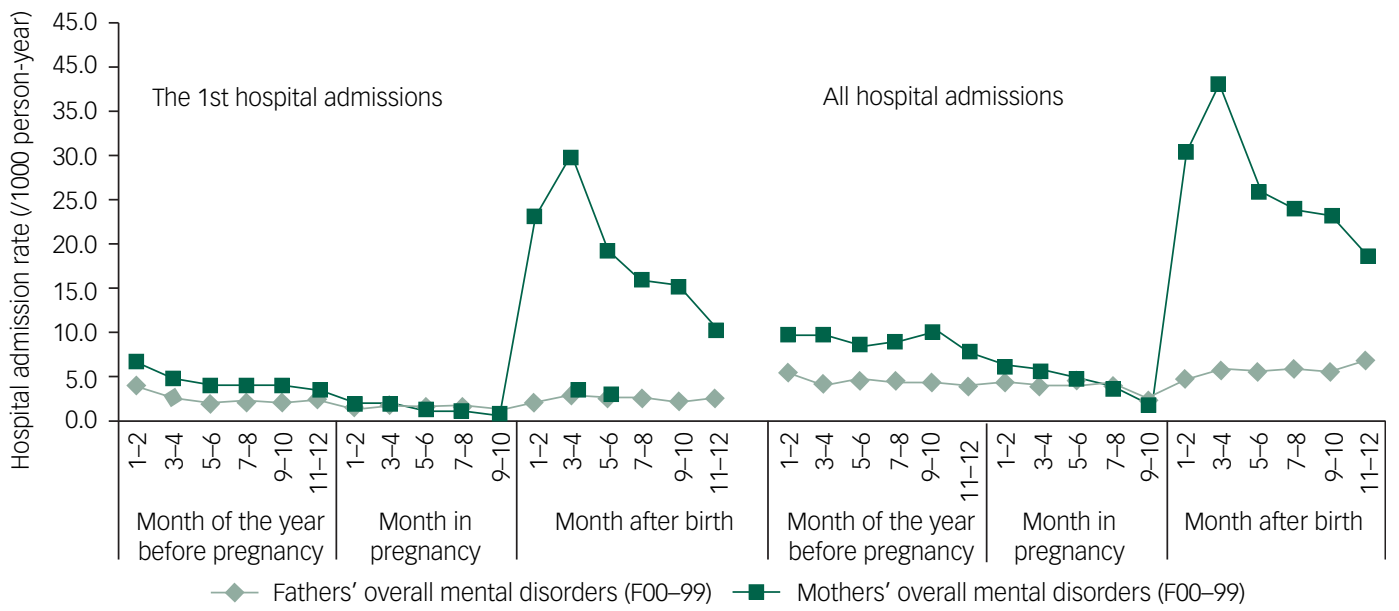

Fig. 3 Couple's hospital admissions for the principal diagnoses of mental disorders over the years before pregnancy, pregnancy and the first year after birth in NSW, Australia, 2001-2010. 


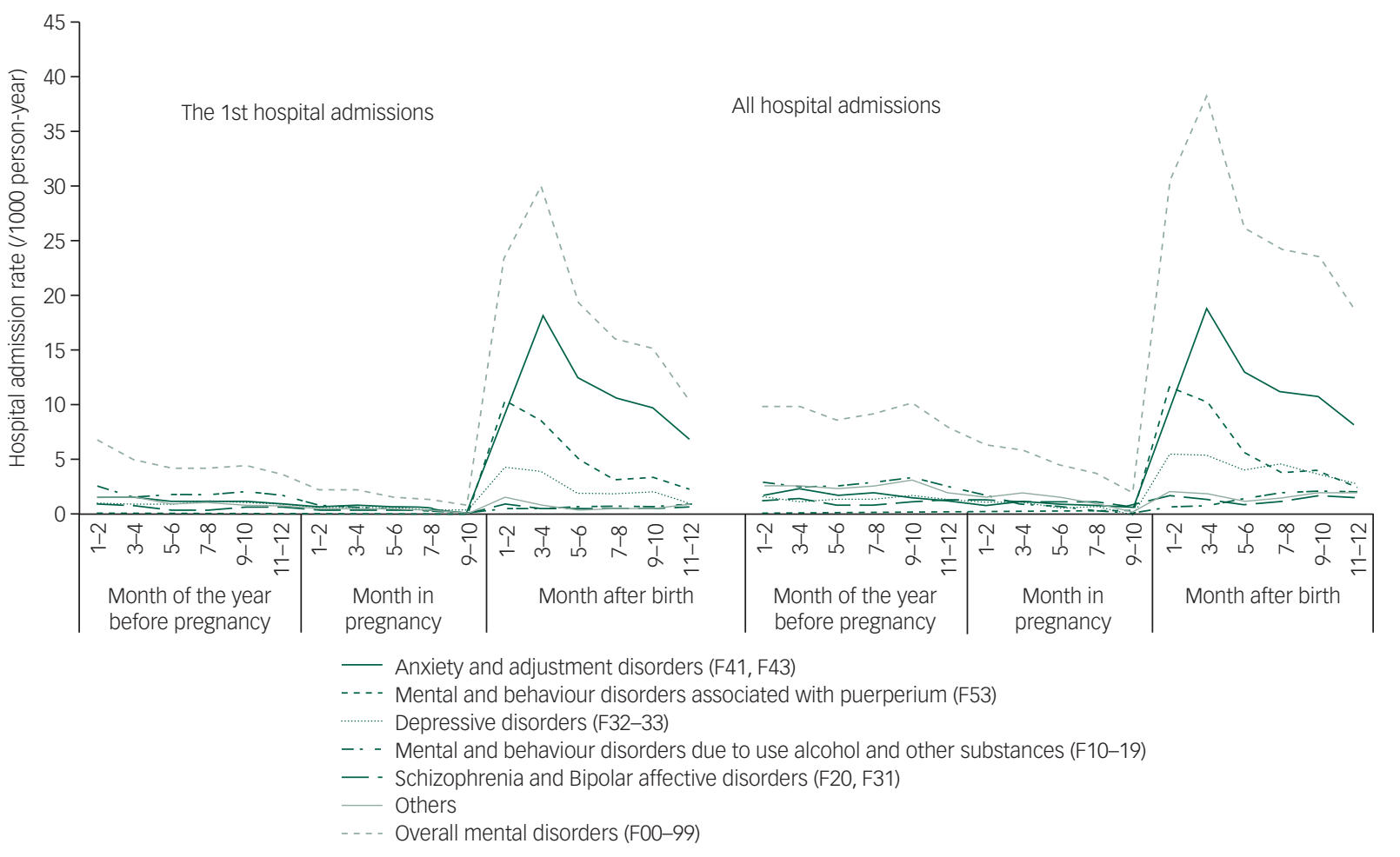

Fig. 4 Mothers' hospital admissions for the principal diagnoses of mental disorders before and after birth in NSW, Australia, 2001-2010. PY person-year; NSW, New South Wales.

no significant difference in the first hospital admission rates for mental disorders between expectant parents.

The increased trend in maternal hospital admissions for mental disorders after birth is well documented in the literature. ${ }^{4,6,7,9}$ The hospital admission rates varied over a wide range. ${ }^{6,9}$ The results of this study are consistent with our previous study which reported that mother's first hospital admission rate for the principal diagnoses of psychiatric disorders (excluding substance use disorders, F10-19) in the first year after birth was 1.67\% (95\% CI 1.63-1.71). ${ }^{9}$ This study showed that mother's first hospital admission rate for mental disorders (including substance use disorders, F10-19) was 1.89\% (95\% CI 1.83-1.95) in the first year after birth (Table 2). A Danish population-based cohort study (1973-2005) also showed that the rate of mother's first-time hospital admission for mental disorders was significantly higher than during pregnancy but the rate levels (the first hospital

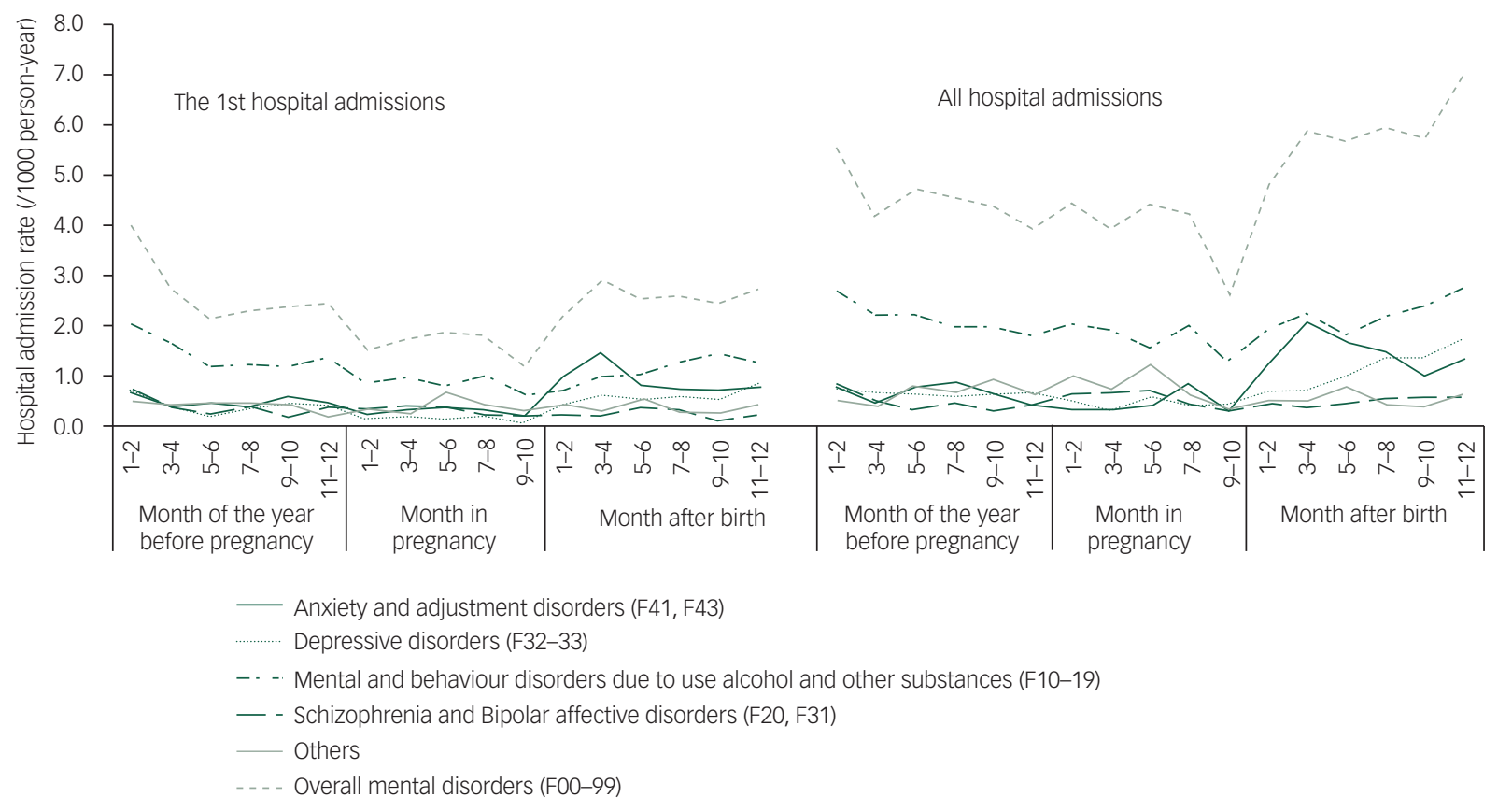

Fig. 5 Fathers' hospital admissions for the principal diagnoses of mental disorders before and after birth in NSW, Australia, 2001-2010. PY person-year; NSW, New South Wales. 


\begin{tabular}{|c|c|c|c|c|c|c|c|c|}
\hline \multirow[b]{2}{*}{ Time of admission } & \multirow{2}{*}{$\begin{array}{l}\text { Fathers with } \\
\text { mental } \\
\text { disorders } \\
\text { (person-year) }\end{array}$} & \multicolumn{3}{|c|}{$\begin{array}{l}\text { Co-occurrence of mothers } \\
\text { with mental disorders }\end{array}$} & \multirow{2}{*}{$\begin{array}{l}\text { Fathers without } \\
\text { mental } \\
\text { disorders } \\
\text { (person-year) }\end{array}$} & \multicolumn{3}{|c|}{$\begin{array}{l}\text { Co-occurrence of mothers } \\
\text { with mental disorders }\end{array}$} \\
\hline & & Mothers & $\%$ & $95 \% \mathrm{Cl}$ & & Mothers & $\%$ & $95 \% \mathrm{Cl}$ \\
\hline Before pregnancy & 524 & 60 & 11.45 & $8.72-14.18$ & 196145 & 861 & 0.44 & $0.35-0.53$ \\
\hline In pregnancy & $195^{\mathrm{a}}$ & 22 & 11.28 & $6.84-15.72$ & 158708 & 234 & 0.15 & $0.09-0.21$ \\
\hline After pregnancy & 503 & 65 & 12.92 & $9.99-15.85$ & 196166 & 3654 & 1.86 & $1.67-2.05$ \\
\hline Overall & $1222^{\mathrm{a}}$ & 147 & 12.03 & $10.21-13.85$ & 551019 & 4749 & 0.86 & $0.78-0.94$ \\
\hline
\end{tabular}

admission rate $0.70 / 1000$ person-year in pregnancy and 1.96/1000 person-year after birth) were significantly lower than the current study. ${ }^{6}$ The increased hospital admissions after birth were mainly attributed to an increase in mother's anxiety and adjustment disorders, mental and behaviour disorders associated with puerperium, and depression (Fig. 4). ${ }^{6,9}$ Alternatively, a survey in the United States showed that there were no significant differences in the prevalence of psychiatric disorders between pregnant (25.3\%), post-partum (27.5\%) and non-pregnant women of child-bearing age $(30.1 \%){ }^{5}$ A retrospective cohort study based on Western Australia health service data between 1990 and 2005 showed that the maternal hospital admission rate for mental disorders in the 12 months before birth was between 14 per 1000 birth (1990) and 17 per 1000 birth (2005), ${ }^{24}$ which was about 9-11 times higher than the expectant mothers' hospital admission rates for mental disorders in this study. The variation of the hospital admission rates for mental disorders may attribute to the difference in study population and time. For example, the study in Denmark was based on the data between 1973 and 2005. ${ }^{6}$ The study in Western Australia was based on the data between 1990 and $2005 .^{24}$ Our study was based on the data from 2003 to 2009. The study from Australia showed that the hospital admission rates for mental disorders increased significantly over the past decade. ${ }^{9,24}$ Other factors, such as the accessibility to health service, ${ }^{25}$ physical health, ${ }^{8}$ location of residence, ${ }^{26}$ country of birth ${ }^{7}$ and maternal age, ${ }^{7,24}$ also impact the hospital admission rate for mental disorders.

The change of paternal hospital admissions for mental disorders before and after birth was not as significant as the rate for mothers. The paternal first hospital admissions for mental disorders did not increase significantly after birth compared with the year before pregnancy. The rate of men's all hospital admissions for mental disorders after birth increased slightly above that of non-fathers during the period of pregnancy and the year before pregnancy. This was attributed to the increase of father's anxiety and adjustment disorders, and depression after birth (Fig. 5). The result of this study was different to the report from Denmark which showed that new father's hospital admission rate for mental disorders in the first year after birth (rate 1.27 per 1000 person-year) was lower than non-fathers (rate 2.08 per 1000 person-year). ${ }^{6}$ The difference between the study in Denmark and our study may be because of different definitions of non- fathers. In the study of Denmark, non-fathers and fathers were different individuals. In our study, non-fathers and fathers were the same individuals in different periods.

A meta-analysis which was based on survey data showed that the depression rate of men in the 2nd quarter of the year after birth (rate 26\%, 95\% CI 17-36\%) was significantly higher than the period of pregnancy and remaining 9 months of the year after birth (rate $11 \%, 95 \%$ CI $6-18 \%$, in the 1 st and 2 nd trimester of gestation; rate $12 \%, 95 \%$ CI $9-15 \%$, in the 3rd trimester of gestation; rate $8 \%, 95 \%$ CI $5-11 \%$, on the 1 st quarter of the year after birth; and rate 9\%, 95\% CI 5-15\%, between the 3rd and 4th quarter). ${ }^{27}$ A longitudinal population-based study of American fathers found that father's depressive symptom scores increased significantly (68\%) in early fatherhood ( $0-5$ years after having the child). ${ }^{14}$ A cohort study of 622 expectant fathers in Hong Kong showed that fathers were more likely to experience depression (EPDSA 13) at 6 weeks post-partum (5.2\%) than early pregnancy (3.3\%) and late pregnancy (4.1\%). ${ }^{28}$ A study in Portugal reported that more fathers experienced mental disorders in the first year after birth than pregnancy. ${ }^{29}$ A cohort study in 5969 adults aged 18-44 in the United States showed that the rate of depression was lower in men than women. ${ }^{30}$

Our study showed that both expectant parents were less likely to be admitted to hospital for the principal mental disorder diagnoses compared with non-parents. The result was consistent with the report which was based on Danish health service data. ${ }^{6}$ The main reason for the decrease was the decline of maternal and paternal hospital admissions for mental and behaviour disorders due to alcohol and substance use disorders during pregnancy (Figs. 4 and 5). A study from the United States reported that pregnant women had significantly lower rates of alcohol and substance use disorders than non-pregnant women. ${ }^{5}$ Our previous study showed that women's hospital admission rate for alcohol use disorders was 1.76 per 1000 person-year (95\% CI 1.45-2.07) before pregnancy and the rate decreased to 0.49 per 1000 personyear (95\% CI $0.36-0.63$ ) during pregnancy and to 0.82 per 1000 person-year (95\% CI $0.67-0.97$ ) in the first year after birth. ${ }^{31}$ However, studies based on community surveys, using self-report screening tools as the measure of mental disorders rather than reported diagnosis, did not show the decrease of mental disorders during pregnancy. ${ }^{12,32}$

\begin{tabular}{|c|c|c|c|c|c|c|c|c|}
\hline \multirow[b]{2}{*}{ Time of admission } & \multirow{2}{*}{$\begin{array}{l}\text { Mothers with } \\
\text { mental disorders } \\
\text { (person-year) }\end{array}$} & \multicolumn{3}{|c|}{$\begin{array}{l}\text { Co-occurrence of fathers } \\
\text { with mental disorders }\end{array}$} & \multirow{2}{*}{$\begin{array}{l}\text { Mothers without } \\
\text { mental disorders } \\
\text { (person-year) }\end{array}$} & \multicolumn{3}{|c|}{$\begin{array}{l}\text { Co-occurrence of fathers } \\
\text { with mental disorders }\end{array}$} \\
\hline & & Fathers & $\%$ & $95 \% \mathrm{Cl}$ & & Fathers & $\%$ & $95 \% \mathrm{Cl}$ \\
\hline Before pregnancy & 921 & 68 & 7.38 & 5.69-9.07 & 195748 & 456 & 0.23 & $0.16-0.30$ \\
\hline In pregnancy & $189^{a}$ & 9 & 4.75 & $1.72-7.78$ & 158714 & 251 & 0.16 & $0.10-0.22$ \\
\hline After pregnancy & 3719 & 70 & 1.88 & $1.44-2.32$ & 192950 & 433 & 0.22 & $0.15-0.29$ \\
\hline Overall & $4829^{a}$ & 147 & 3.04 & $2.56-3.52$ & 547412 & 1140 & 0.21 & $0.17-0.25$ \\
\hline
\end{tabular}


The results of this study found that parents' mental disorders influenced each other, and women were more likely to be impacted by their partner's mental health problems compared with men. A study based on community data from England showed that men's depressive symptoms were correlated with their partners' depressive symptoms before $(r=0.24)$ and after $(r=0.26)$ the birth. ${ }^{16} \mathrm{~A}$ cross-sectional study from Italy found that maternal distress was significantly associated with paternal distress $(r=0.486) .{ }^{33}$ A study in Japan reported that father's depression was impacted by partner's depression (adjusted odds ratio 1.91, 95\% CI 1.05-3.47). ${ }^{34}$ An integrative review showed that maternal depression was a strong predictor of paternal depression during the post-partum period. ${ }^{13}$

The strength of this study is that the study data are relatively complete (all new parents in NSW were consecutively followed up for 3 years, from non-parents, expectant parents to parents) and accurate (women and men were paired by birth). As a result, both maternal and paternal hospital admission rates can be described consecutively through the stages of non-parents, expectant parents to parents. This allows a comparison of rates between men and women, and between non-parents, expectant parents and parents in hospital admissions with mental disorders. The data also allowed us to examine the impact of maternal and paternal mental disorders on each other.

There are some limitations that need to be considered when interpreting the results of this population study. The study neither includes epigenetic data nor data on community and out-patient mental health services, which may be differentially accessed by both populations and impact rates of hospital admission. Second, some researchers have suggested that hospital admissions for mental disorders may be over-enumerated because admissions could occur for medical reasons associated with the perinatal period. ${ }^{35,36}$ To minimise the potential overestimation of incidence rates, we included only those admissions with a 'principal' diagnosis of mental disorder. Birth registration data describe the family structure at the time the birth was registered, which is not necessarily the family structure at the time of the birth. The baby's father on the RBDM birth registration file was not necessarily the biological father. The data did not allow us to examine the impact of biological and non-biological fathers' mental disorders on mothers respectively.

The incidence rate of mothers' mental disorders after birth increased more significantly than the fathers' rate. There was an association between mother's mental disorders and father's mental disorders suggesting that development and testing of parents-based intervention would be important to explore to address family well-being.

Fenglian Xu, PhD, Faculty of Health, University of Technology Sydney, Ultimo, Australia; Elizabeth Sullivan, MD, Faculty of Health, University of Technology Sydney, Ultimo, Australia; Colin Binns, PhD, School of Public Health, Curtin University, Perth, Australia; Caroline S. E. Homer, PhD, Faculty of Health, University of Technology Sidney, Ultimo, Australia

Correspondence: Fenglian Xu, Faculty of Health, University of Technology Sydney, Ultimo, Australia. Email: Fenglian.Xu@uts.edu.au

First received 3 Feb 2016, final revision 2 May 2016, accepted 16 May 2016

\section{Acknowledgements}

We would like to thank data custodians of the Ministry of Health and staff of the Centre for Health Record Linkage (CheReL) for providing the data, undertaking data linkage and providing advice. We acknowledge the families who have contributed their data and professional staff who were involved in the data collection and management for this research.

\section{References}

1 Almond P. Postnatal depression: a global public health perspective. Perspect Public Health 2009; 129: 221-7.

2 World Health Organization. Mental Health Aspects of Women's Reproductive Health: A Global Review of the Literature. WHO Library, 2009.

3 Wisner KL, Chambers C, Sit DK. Postpartum depression: a major public health problem. JAMA 2006; 296: 2616-8.

4 Kendell RE, Chalmers JC, Platz C. Epidemiology of puerperal psychoses. Br J Psychiatry 1987; 150: 662-73.

5 Vesga-Lopez O, Blanco C, Keyes K, Olfson M, Grant BF, Hasin DS. Psychiatric disorders in pregnant and postpartum women in the United States. Arch Gen Psychiatry 2008; 65: 805-15.

6 Munk-Olsen T, Laursen TM, Pedersen CB, Mors O, Mortensen PB. New parents and mental disorders: a population-based register study. JAMA 2006; 296: 2582-9.

7 Xu F, Austin MP, Reilly N, Hilder L, Sullivan EA. Major depressive disorder in the perinatal period: using data linkage to inform perinatal mental health policy. Arch Womens Ment Health 2012; 15: 333-41.

8 Woolhouse $\mathrm{H}$, Gartland D, Perlen S, Donath S, Brown SJ. Physical health after childbirth and maternal depression in the first 12 months post partum: results of an Australian nulliparous pregnancy cohort study. Midwifery 2014; 30: 378-84.

9 Xu F, Sullivan EA, Li Z, Burns L, Austin MP, Slade T. The increased trend in mothers' hospital admissions for psychiatric disorders in the first year after birth between 2001 and 2010 in New South Wales, Australia. BMC Womens Health 2014; 14: 119.

10 Munk-Olsen T, Laursen T, Mendelson T, Pedersen C. Perinatal mental disorders in native Danes and immigrant women. Arch Womens Ment Health 2010; 13: 319-26.

11 Ibanez G, Blondel B, Prunet C, Kaminski M, Saurel-Cubizolles MJ. Prevalence and characteristics of women reporting poor mental health during pregnancy: findings from the 2010 French National Perinatal Survey. Rev Epidemiol Sante Publique 2015; 63: 85-95.

12 Milgrom J, Gemmill AW, Bilszta JL, Hayes B, Barnett B, Brooks J, et al. Antenatal risk factors for postnatal depression: a large prospective study. J Affect Disord 2008; 108: 147-57.

13 Goodman JH. Paternal postpartum depression, its relationship to maternal postpartum depression, and implications for family health. J Adv Nurs 2004; 45: 26-35.

14 Garfield CF, Duncan G, Rutsohn J, McDade TW, Adam EK, Coley RL, et al. A longitudinal study of paternal mental health during transition to fatherhood as young adults. Pediatrics 2014; 133: 836-43.

15 Wee KY, Skouteris H, Pier C, Richardson B, Milgrom J. Correlates of ante- and postnatal depression in fathers: a systematic review. J Affect Disord 2011; 130: 358-77.

16 Deater-Deckard K, Pickering K, Dunn JF, Golding J. Family structure and depressive symptoms in men preceding and following the birth of a child. The Avon Longitudinal Study of Pregnancy and Childhood Study Team. Am J Psychiatry 1998; 155: 818-23.

17 Pinheiro RT, Magalhaes PV, Horta BL, Pinheiro KA, da Silva RA, Pinto RH. Is paternal postpartum depression associated with maternal postpartum depression? Population-based study in Brazil. Acta Psychiatr Scand 2006; 113: 230-2.

18 Edward KL, Castle D, Mills C, Davis L, Casey J. An integrative review of paternal depression. Am J Mens Health 2015; 9: 26-34.

19 New South Wales Government. Birth: Registry of Births, Deaths and Marriages, 2015 (http://www.bdm.nsw.gov.au/Pages/births/births.aspx).

20 National Centre for Classification in Health. The International Statistical Classification of Diseases and Related Health Problems, 10th Revision, Australian Modification (ICD-10-AM). National Centre for Classification in Health, 1999.

21 Department of Health Centre for Health Record Linkage. How Record Linkage Works, 2016 (http://www.cherel.org.au/how-record-linkage-works).

22 Australian Institute of Health and Welfare. Australian Hospital Statistics 2008-09. Health Services series no. 17. Cat. no. HSE 84. AlHW, 2010

23 IBM. SPSS Software, 2015. (http://Www-01.ibm.com/software/analytics/spss/).

24 O'Donnell M, Anderson D, Morgan VA, Nassar N, Leonard HM, Stanley FJ. Trends in pre-existing mental health disorders among parents of infants born in Western Australia from 1990 to 2005. Med J Aust 2013; 198: 485-8.

25 The Department of Health. Key Findings from the Program Evaluation of the Better Access to Psychiatrists, Psychologists and General Practitioners through the Medicare Benefits Schedule Initiative: Fact Sheet, 2015. (http://www.health.gov. au/internet/main/publishing.nsf/Content/mental-ba-eval-fact).

26 Buist $A E$, Austin MP, Hayes BA, Speelman C, Bilszta JL, Gemmill AW, et al. Postnatal mental health of women giving birth in Australia 2002-2004: findings 
from the beyondblue National Postnatal Depression Program. Aust $N$ Z J Psychiatry 2008; 42: 66-73.

27 Paulson JF, Bazemore SD. Prenatal and postpartum depression in fathers and its association with maternal depression: a meta-analysis. JAMA 2010; 303: 1961-9.

28 Koh YW, Chui CY, Tang CS, Lee AM. The prevalence and risk factors of paternal depression from the antenatal to the postpartum period and the relationships between antenatal and postpartum depression among fathers in Hong Kong. Depress Res Treat 2014; 2014: 127632.

29 Areias ME, Kumar R, Barros H, Figueiredo E. Comparative incidence of depression in women and men, during pregnancy and after childbirth. Validation of the Edinburgh Postnatal Depression Scale in Portuguese mothers. Br J Psychiatry 1996; 169: 30-5.

30 Anthony JC, Petronis KR. Suspected risk factors for depression among adults 18-44 years old. Epidemiology 1991; 2: 123-32.

31 XU F, Bonello M, Burns L, Austin MP, Li Z, Sullivan E. Hospital admissions for alcohol use disorders before, during, and after pregnancy: a study based on linked population data in new South Wales, Australia. Alcohol Clin Exp Res 2013; 37: 1706-12.

32 O'Hara MW, Wisner KL. Perinatal mental illness: definition, description and aetiology. Best Pract Res Clin Obstet Gynaecol 2014; 28: 3-12.
33 Epifanio MS, Genna V, De Luca C, Roccella M, La Grutta S. Paternal and maternal transition to parenthood: the risk of postpartum depression and parenting stress. Pediatr Rep 2015; 7: 5872

34 Nishimura A, Fujita $Y$, Katsuta $M$, Ishihara A, Ohashi K. Paternal postnatal depression in Japan: an investigation of correlated factors including relationship with a partner. BMC Pregnancy Childbirth 2015; 15: 128

35 Jones I, Heron J, Blackmore ER, Craddock N. Incidence of hospitalization for postpartum psychotic and bipolar episodes. Arch Gen Psychiatry 2008; 65: 356.

36 Matthey S, Ross-Hamid C. The validity of DSM symptoms for depression and anxiety disorders during pregnancy. J Affect Disord 2011; 133: 546-52. 